\title{
Percepciones sobre Educación Medioambiental y Vial en futuros docentes del Máster en Profesorado Educación Secundaria
}

\section{Environmental and Road Safety Education perceptions for future tutors of master's degree in Educación Secundaria (junior high school) Teaching}

\author{
Francisco Manuel Morales Rodríguez*, Manuel Alejandro Narváez Peláez * Juan Ramón Pereira Docampo** \\ *Universidad de Málaga, ** Asociación por la divulgación, investigación y docencia de la psicología
}

\begin{abstract}
Resumen
En una de las actividades prácticas en la que se ha clarificado la importancia de los temas transversales en Educación Secundaria, se ha aplicado a 54 alumnos y alumnas de la asignatura Aprendizaje y desarrollo de la personalidad del Máster en Profesorado de Educación Secundaria la adaptación de un instrumento para analizar percepciones, actitudes e intereses respecto a la Educación Ambiental y Vial, que constituyen uno de los temas transversales más relevantes en nuestro contexto. Se concluye señalando la utilidad e importancia de estos datos para detectar posibles sesgos, estereotipos y percepciones de los futuros docentes respecto a estos temas transversales.

Palabras clave: universitarios, Educación Ambiental, Educación Vial
\end{abstract}

\begin{abstract}
One of the practical activities, proved to be essential for the transversal subjects in Educación Secundaria (junior high school), have been handed out to 54 postgraduate students of "Aprendizaje y Desarrollo de la Personalidad" (Learning and Personality Development), from the master's degree in "Educación Secundaria (junior high school) Teaching". It consists in an adaptation tool that analyse perceptions, attitudes and interests regarding Environmental and Road Safety Education, which is considered one of the most notable subjects in this context The study ends off pointing out the importance of this information in order to detect possible slants, stereotypes and perceptions of the future teachers regarding this transversal subjects.

Keywords: university students, Environmental Education, Road Safety Education
\end{abstract}

El presente trabajo se enmarca en el Proyecto de Innovación Educativa (PIE13-031) que tiene entre sus objetivos fomentar y evaluar competencias transversales fundamentales para la formación del alumnado, que se adquieren a través de la realización de actividades prácticas. En este sentido, en una de las actividades prácticas en la que se ha clarificado la importancia de los temas transversales en Educación Secundaria, se ha pasado a 54 alumnos y alumnas de la asignatura Aprendizaje y desarrollo de la personalidad del Máster en Profesorado de Educación Secundaria la adaptación de un instrumento para analizar percepciones, actitudes e intereses respecto a la Educación Ambiental y Vial, que constituyen uno de los temas transversales más relevantes en nuestro contexto.
Este trabajo es continuidad de varios proyectos de innovación educativa en los que se ha participado con el objetivo de fomentar competencias transversales como las sociales y ciudadanas.

Una línea emergente en nuestra sociedad y en los planes estratégicos de las Universidades es la relacionada con el fomento de competencias transversales para la protección y educación del medio ambiente. De la misma manera que en otros proyectos se ha enfatizado la importancia de la solidaridad cotidiana, en este caso también puede hablarse de una solidaridad relacionada con la protección del medio ambiente en la vida cotidiana en el ámbito universitario; en coherencia y/o dotando de continuidad lo que se propone en algunos trabajos para otros niveles educativos (Massanés y Miralles, 1995; Pro, 2014)

Entre los desafíos ambientales que hay que afrontar se encuentran los relacionados con los desequilibrios demográficos (solidaridad intergeneracional y crisis de las pensiones, etc), la energía, la pérdida de cubierta vegetal, la cuestión alimentaria, la contaminación, los conflictos bélicos y la destrucción ambiental (Novo, 1999); y el agua y el cambio climático (Novo, 1999; Sempere y Riechmann, 2004).

Es necesario evaluar y fomentar actitudes y comportamientos respetuosos con el medio ambiente considerando la importancia del estudio del comportamiento proambiental (Corral, 2001) y del cada vez mayor número de propuestas que pueden encontrarse en la literatura para la toma de conciencia y desarrollo de acciones proambientales (Damin y Monteleone, 2002; De Castro, 2000; Dobson, 1997; McHarry, 1994) y para la educación vial (Álvarez, González y Robledo, 2004; Dirección General de Tráfico, 1994; Dirección General de Tráfico, 1996).

El objetivo general del presente trabajo es aportar los resultados iniciales de aplicar un Cuestionario para profesorado sobre Educación Medioambiental y Vial.

\section{Método}

\section{Participantes}

Los participantes son 54 alumnos y alumnas de la asignatura Aprendizaje y desarrollo de la personalidad del Máster en Profesorado de Educación Secundaria, de 
la Especialidad de TIPI de la provincia de Málaga. El $64 \%$ son hombres y con edades comprendidas entre 24 y 49 años (el 12.7\% tiene 28 años).

\section{Instrumentos}

Se ha utilizado para evaluar las actitudes y percepciones relacionadas con la Educación Vial y Ambiental los siguientes instrumentos:

1) Adaptación del Cuestionario para Profesores Sobre Educación Ambiental (Martín, León, Margalef y Pardo, 2001; citado en Martín, 2001). Este instrumento incluye distintos tipos de preguntas. En algunas de ellas se solicita al alumnado que marque el grado con el que se identifica con una serie de afirmaciones según escala tipo Likert de cinco puntos de menor a mayor grado de identificación.

2) Adaptación del Cuestionario para profesores para Educación Vial (Martín, León, Margalef y Pardo, 2001; citado en Martín, 2001). La estructura y el formato son muy similares al anterior pero centrado de forma más concreta y específica en aspectos y cuestiones relacionados con la Educación Vial. Incluye respuestas tipo Likert de 5 opciones: A, B, C, D, E.

\section{Procedimiento}

Los datos se aplicaron en el grupo de clase y se aseguró el carácter voluntario de la participación y tanto la confidencialidad de la información como el hecho de que no se comparará por grupos con un tratamiento global de los datos sin particularizar ni comparar entre personas o grupos.

\section{Resultados}

Entre los resultados puede destacarse que el 76.6\% procura como profesor que en las diferentes tareas y actividades se promueva en el alumnado un verdadero cambio de actitudes;

El $66 \%$ considera que la Educación Ambiental se contempla como tema transversal en la medida que es posible con el trabajo de cuestiones ambientales y áreas y materias que más se prestan a colaborar;

El $61.7 \%$ señala que solo algunas veces, durante el curso, cuando surge una ocasión propicia se promueven en el aula estrategias para la enseñanza de valores ambientales, por ejemplo, mediante dilemas morales, etc.

Con respecto a la Educación Vial, los porcentajes más elevados se han obtenido en estos aspectos:

El $82.20 \%$ está totalmente de acuerdo con la afirmación de que "la Educación Vial es necesaria para el desenvolvimiento del ciudadano en la sociedad";

El $61.4 \%$ considera que este contenido debe desarrollarse como contenido transversal;

El $75 \%$ y el $72.7 \%$ señalan que en el centro deben desarrollarse aún más temas como el coche y el ciclomotor respectivamente;

El $91 \%$ de los futuros docentes percibe que no tiene formación para recibir esta materia;

El $46.7 \%$ otorga una importancia de 3 (según escala de 1 a 5) al grado en que la Educación Vial está presente en el Centro en las dimensiones objetivos específicos y en las actividades escolares;
El 40\% manifiesta que solo algunas veces en el aula promueven estrategias para la enseñanza de la Educación Vial, entendida como un valor;

El 37.8\% y el 42.2\% respectivamente están bastante de acuerdo con las afirmación de que "La Educación Vial contribuye a fomentar actitudes positivas en los alumnos" y "por medio de la Educación Vial se pueden desarrollar hábitos de comportamiento en el alumnado", etc. independientemente de su orientación sexual, sexo, raza, condición física", etc) y el respeto a espacios reservados para ancianos, etc.

$\mathrm{Si}$ realizamos un análisis DAFO (Debilidades, Amenazas, Fortalezas y Oportunidades) podemos destacar que en los debates y discusiones que se han realizado, las actividades educativas para la mejora de la cultura medioambiental y vial, despiertan bastante interés en los futuros docentes y en el alumnado que son los auténticos protagonistas del proceso de enseñanza-aprendizaje en el Espacio Europeo de Educación Superior.

\section{Conclusiones}

Se concluye señalando la utilidad e importancia de estos datos para detectar posibles sesgos, estereotipos y percepciones de los futuros docentes respecto a estos temas transversales que están cobrando cada vez más relevancia en Educación Secundaria tratando de contribuir a la mejora de calidad de la formación relacionada con estos aspectos.

Los resultados ponen de manifiesto que para los futuros docentes se trata de una línea temática muy relevante, en la que es necesario aunar esfuerzos que contribuyan a la mejora de la calidad educativa relacionada con la Educación Ambiental y Vial.

Compartimos la percepción con los participantes en este trabajo de la importancia de asumir como docentes la responsabilidad social y la necesidad de incluir actividades y propuestas pedagógicas para la mejora formativa y toma de conciencia relacionada con los problemas, retos y desafíos propios de la Educación Medioambiental y Vial. Entre esos desafíos pueden encontrarse el problema de la insostenibilidad ambiental (Folch, 1998). Para Folch (1998) en su interesante libro titulado "Ambiente, Emoción y Ética. Actitudes ante la cultura de la sostenibilidad" en este ámbito uno de los interrogantes está en el hecho de que: “...los valores éticos acaban siendo simples referentes estéticos" ( $\mathrm{p}$. 51).

Nuestra intención es dotar de continuidad esta aproximación inicial a este ámbito y convertirlo en una de las posibles líneas de trabajo e innovación educativa en nuestro contexto.

\section{Referencias}

Álvarez, F. J., González, E. y Robledo, T. (2004). Cuaderno didáctico sobre Educación Vial y Salud. Madrid: CIDE.

Corral, V. (2001). Comportamiento proambiental. Introducción al estudio de las conductas protectoras del ambiente. Santa Cruz de Tenerife: Editorial Resma, S. L. 
Damin, R. y Monteleone, A. (2002). Temas ambientales en el aula. Barcelona: Paidós.

De Castro, R. (2000). Voluntariado ambiental. Claves para la acción ambiental comunitaria. Illes Balears: Di 7 Edicó.

Dirección General de Tráfico. (1994). Educación Vial. Temario destinado a la enseñanza en las Escuelas Universitarias de Formación del Profesorado. Madrid: Dirección General de Tráfico.

Dirección General de Tráfico. (1996). Educación Vial. 10 años de Investigación para la Educación Vial. Madrid: Dirección General de Tráfico.

Dobson, A. (1997). Pensamiento político verde. Una nueva ideología para el siglo XXI. Barcelona: Ediciones Paidós Ibérica, S. A.

Folch, R. (1998). Ambiente, emoción y ética. Actitudesante la cultura de la sostenibilidad. Barcelona: Ediciones Ariel, S. A.
Martín, M. (Coord.) (2001). Una reflexión en torno a los contenidos transversales: Educación Ambiental y Educación Vial. Madrid: Universidad de Alcalá.

Massanés, R. y Miralles, J. (1995). Ecología de cada día. Barcelona: Naturart, S. A.

McHarry, J. (1994). Reducir, Reutilizar, Reciclar. Madrid: A.M. Editor.

Novo, M. (Coord.). (1999). Los desafios ambientales. Reflexiones y propuestas para un futuro sostenible. Madrid: Editorial Universitas, S. A.

Pro, A. (2014). La energía: Uso, Consumo y Ahorro energético en la vida cotidiana. Barcelona: Editorial GRAÓ.

Sempere, J. y Riechmann, J. (2004). Sociología y Medio Ambiente. Madrid: Editorial Síntesis. 